\title{
ANALYSIS OF A CLASS OF NONLINEAR INTEGRO-DIFFERENTIAL EQUATIONS ARISING IN A FORESTRY APPLICATION
}

\author{
BY \\ MICHAEL A. KRAEMER AND LEONID V. KALACHEV \\ Department of Mathematical Sciences, University of Montana, Missoula, MT 59821
}

\begin{abstract}
Certain models describing the age dynamics of a natural forest give rise to nonlinear integro-differential equations for the seedlings density as a function of time. The special feature of the problem is that corresponding solutions have non-smooth second derivatives. Since the biological model contains a small parameter, a perturbation method can be used to find an asymptotic solution. Banach's fixed point theorem is used to prove existence and uniqueness of the solution, the convergence of a numerical scheme, and the validity of the asymptotic approximation. In an example numerical and asymptotic approximations are compared for various choices of time steps.
\end{abstract}

1. Introduction. We consider a class of models where tree seedlings become established on a site following a non-catastrophic disturbance that eliminates the tree canopy. They will grow freely until the onset of competition for growing space with neighboring trees. Growing space can be thought of as the intangible measure of a plant's capacity to grow until one of the factors necessary for growth (i.e., a site resource) becomes limiting (see [5], [7]). In [4] quasilinear partial differential equations were used to model the density of trees in a natural forest as a function of time and tree age. With a suitable function describing the mortality of trees depending on tree age and stand density, the method of characteristics can be used to reduce the partial differential equation to an integro-differential equation for the seedlings density as a function of time, the so-called seedlings equation. Setting the time derivative in the seedlings equation to zero results in a nonlinear integral equation for the seedlings quasi-equilibrium, that is, the number of seedlings that survive to become trees. Since competition from other trees changes with time according to an increase or decrease of the biomass of the forest as a whole, this quasi-equilibrium is time-dependent. It is approached with a delay called seedlings

Received October 10, 2001.

2000 Mathematics Subject Classification. Primary 45J05, 34E10.

E-mail address: kraemer@selway.umt.edu

E-mail address: leonid@selway.umt.edu 
re-establishment time. The rescaled seedlings equation contains a small parameter $\varepsilon$, the ratio of seedlings re-establishment time and the average lifespan of a tree.

In this paper we use a unifying approach, Banach's fixed point theorem, to establish existence and uniqueness of the solution of the problem, convergence of a numerical scheme for solving the problem, and for proving the theorem on estimation of the remainder term for asymptotic approximation of the solution of the problem. These results provide the mathematical basis for the next steps of the analysis of age structure models related to explicit determination of functions and parameters entering the models by fitting the solutions to data from real field measurements using nonlinear least squares methods. Results of such nonlinear least squares fitting procedures applied to particular age structure models will be published elsewhere.

The paper is organized as follows: In Sec. 2 we formulate the initial value problem for the seedlings density, and present the integral equation for the quasi-equilibrium solution. In Sec. 3 we prove existence and uniqueness of the solution of both abovementioned problems and derive some of their properties. Linear convergence of the numerical solution is shown in Sec. 4. Section 5 contains the description of the algorithm for construction of an asymptotic solution using the boundary function method and the estimation of the remainder, which is the proof that the norm of the difference of asymptotic solution and exact solution is of order $O(\varepsilon)$. Some auxiliary results can be found in the Appendix.

2. Mathematical model. For a particular class of models introduced in [4], the integro-differential equation for seedlings density $s(t)$ as a function of time, has the (rescaled) form

$$
\begin{aligned}
\varepsilon s^{\prime}(t) & =-s(t)+\max (0,1-\lambda v(t)), \\
v(t) & =v_{\text {old }}(t)+\int_{0}^{t} b(a) e^{-a} s(t-a) d a, \\
s(0) & =s^{0} .
\end{aligned}
$$

In (1), $0<\varepsilon \ll 1$ is a small parameter, which represents the ratio of seedlings reestablishment time and the average life span of a tree. The competition factor $\lambda$ measures how competition from other seedlings and older trees inhibits the number of seedlings. Function $v(t)$ represents the total volume of the forest as a function of time. The old growth volume $v_{\text {old }}(t)$ describes the total volume of the trees already existing at the initial time, whereas the integral $\int_{0}^{t} b(a) e^{-a} s(t-a) d a$ is the total volume of the trees that grew from seedlings after initial time. The size function $b(a)$ describes the average size of individual trees of age $a$. Depending on the particular model used, the size function represents either height, basal area, stem volume, or crown area. The model assumes a constant relative death rate of trees, which has been rescaled to 1 and is reflected in the factor $e^{-a}$ in the integral in (2). The term $\max (0,1-\lambda v(t))$ corresponds to the seedlings quasi-equilibrium at time $t$, which is approached exponentially as expressed in equation (1). When the forest volume $v(t)$ increases above the critical volume

$$
v_{\text {crit }}=1 / \lambda
$$


the seedlings quasi-equilibrium becomes zero, and seedlings density $s(t)$ decreases exponentially at per-capita rate $1 / \varepsilon$. At the transition point where $v=v_{\text {crit }}$ a discontinuity of the second derivative of the seedlings density occurs.

Let us consider problem (1)-(3) in a finite time interval $0 \leq t \leq T$. With notations

$$
\begin{aligned}
f(a) & =b(a) e^{-a}, \\
g(t) & =1-\lambda v_{\text {old }}(t), \\
K s(t) & =g(t)-\lambda \int_{0}^{t} f(a) s(t-a) d a, \\
K^{+} s(t) & =\max (0, K s(t)),
\end{aligned}
$$

the initial value problem can be written as

$$
\begin{aligned}
\varepsilon s^{\prime}(t) & =-s(t)+K^{+} s(t) \quad \text { for } 0 \leq t \leq T, \\
s(0) & =s^{0}
\end{aligned}
$$

We also analyse the quasi-equilibrium problem associated with (9), (10) which is obtained by replacing the derivative in (9) by zero and omitting condition (10):

$$
0=-s(t)+K^{+} s(t) \text { for } 0 \leq t \leq T .
$$

Assuming that the old growth function $v_{\text {old }}(t)$ and the size function $b(t)$ are continuously differentiable, the functions $f(t)$ and $g(t)$ defined in (5) and (6) are also continuously differentiable, and the operators $K$ and $K^{+}$map the space of continuous functions on the interval $[0, T]$ into itself. This space, which we denote by $C([0, T])$, is a Banach space with the maximum norm

$$
\|s\|=\max _{0 \leq t \leq T}|s(t)|
$$

Note that the solutions of the quasi-equilibrium problem (11) are the fixed points of the operator $K^{+}$in the Banach space $C([0, T])$. In what follows we often utilize the identity

$$
\int_{0}^{t} f(t-a) s(a) d a=\int_{0}^{t} f(a) s(t-a) d a,
$$

which results from the substitution $a \rightarrow t-a$ in the integral.

\section{Existence, uniqueness, and properties of solutions.}

ThEOREM 1. The quasi-equilibrium problem (11) has exactly one solution $s_{q e}^{*}(t)$ in $C([0, T])$. This solution is non-negative, bounded from above by 1 , and satisfies a Lipschitz condition of order 1 with Lipschitz constant

$$
C_{q e}=\left\|g^{\prime}\right\|+\lambda\left\|s_{q e}^{*}\right\|\left(T\left\|f^{\prime}\right\|+\|f\|\right) .
$$

Proof. To show that operator $K^{+}: C([0, T]) \rightarrow C([0, T])$ has a unique fixed point, consider any two functions $s_{1}, s_{2} \in C([0, T])$ and any $t \in[0, T]$. By virtue of (7), (8), 
and Corollary 1 (see Appendix), we have the estimate:

$$
\begin{aligned}
\left|K^{+} s_{1}(t)-K^{+} s_{2}(t)\right| & =\left|\max \left(0, K s_{1}(t)\right)-\max \left(0, K s_{2}(t)\right)\right| \\
& \leq\left|K s_{1}(t)-K s_{2}(t)\right| \\
& \leq \lambda \int_{0}^{t} f(t-a)\left|s_{1}(a)-s_{2}(a)\right| d a \\
& \leq\left\|s_{1}-s_{2}\right\| \cdot \lambda\|f\| t .
\end{aligned}
$$

The rest of the existence and uniqueness proof uses standard techniques for Volterra integral operators (see [1]). By induction over $n$ it can be shown that

$$
\left|\left(K^{+}\right)^{n} s_{1}(t)-\left(K^{+}\right)^{n} s_{2}(t)\right| \leq\left\|s_{1}-s_{2}\right\| \cdot \frac{(\lambda\|f\|)^{n} t^{n}}{n !}
$$

for $n=0,1,2, \ldots$. Since the infinite series

$$
\sum_{n=0}^{\infty} \frac{(\lambda\|f\|)^{n} T^{n}}{n !}=\exp (\lambda\|f\| T)
$$

converges, we conclude that

$$
\lim _{n \rightarrow \infty} \frac{(\lambda\|f\|)^{n} T^{n}}{n !}=0 .
$$

Therefore there exists $N$ such that

$$
\frac{(\lambda\|f\|)^{N} T^{N}}{N !}<\frac{1}{2} .
$$

From (16) it follows that

$$
\left\|\left(K^{+}\right)^{N} s_{1}-\left(K^{+}\right)^{N} s_{2}\right\| \leq \frac{1}{2}\left\|s_{1}-s_{2}\right\| .
$$

Hence $K\left({ }^{+}\right)^{N}: C([0, T]) \rightarrow C([0, T])$ is a contraction mapping. By a corollary to Banach's fixed point theorem, the mapping $K^{+}$has exactly one fixed point $s_{q e}^{*}(t)$ in $C([0, T])$. This fixed point is the unique solution of the quasi-equilibrium problem (11).

The function $s_{q e}^{*}(t)$ is non-negative, since

$$
s_{q e}^{*}(t)=\max \left(0, K\left(s_{q e}^{*}(t)\right)\right) \geq 0 .
$$

It is bounded from above by 1 , since by virtue of (6) and (7),

$$
\begin{aligned}
s_{q e}^{*}(t) & =\max \left(0, K\left(s_{q e}^{*}(t)\right)\right) \\
& \leq K\left(s_{q e}^{*}(t)\right) \\
& =1-\lambda\left(v_{\text {old }}(t)+\int_{0}^{t} f(a) s_{q e}^{*}(t-a) d a\right) \\
& \leq 1 .
\end{aligned}
$$


The fact that $s_{q e}^{*}(t)$ satisfies a Lipschitz condition of order 1 follows from (11) and Corollary 1 (see Appendix). Indeed, for any $t_{1}, t_{2} \in[0, T]$, we have

$$
\begin{aligned}
\left|s_{q e}^{*}\left(t_{1}\right)-s_{q e}^{*}\left(t_{2}\right)\right|= & \left|\max \left(0, K s_{q e}^{*}\left(t_{1}\right)\right)-\max \left(0, K s_{q e}^{*}\left(t_{2}\right)\right)\right| \\
\leq & \left|K s_{q e}^{*}\left(t_{1}\right)-K s_{q e}^{*}\left(t_{2}\right)\right| \\
\leq & \left|g\left(t_{1}\right)-g\left(t_{2}\right)\right| \\
& +\lambda\left|\int_{0}^{t_{1}} f\left(t_{1}-a\right) s_{q e}^{*}(a) d a-\int_{0}^{t_{2}} f\left(t_{2}-a\right) s_{q e}^{*}(a) d a\right| \\
\leq & \left\|g^{\prime}\right\|\left|t_{1}-t_{2}\right| \\
& +\lambda\left|\int_{0}^{t_{2}}\left(f\left(t_{1}-a\right)-f\left(t_{2}-a\right)\right) s_{q e}^{*}(a) d a-\int_{t_{1}}^{t_{2}} f\left(t_{1}-a\right) s_{q e}^{*}(a) d a\right| \\
\leq & \left\|g^{\prime}\right\|\left|t_{1}-t_{2}\right| \\
& +\lambda T\left\|f^{\prime}\right\|\left\|s_{q e}^{*}\right\|\left|t_{1}-t_{2}\right|+\lambda\|f\|\left\|s_{q e}^{*}\right\|\left|t_{1}-t_{2}\right| \\
= & \left(\left\|g^{\prime}\right\|+\lambda\left\|s_{q e}^{*}\right\|\left(T\left\|f^{\prime}\right\|+\|f\|\right)\right)\left|t_{1}-t_{2}\right| .
\end{aligned}
$$

Theorem 2. The initial-value problem (9), (10) has exactly one solution $s^{*}(t)$ in $C^{1}([0, T])$. This solution is non-negative and bounded from above by 1 . Its derivative is bounded in absolute value by $1 / \varepsilon$, and it satisfies a Lipschitz condition of order 1 with Lipschitz constant

$$
C=\frac{\left\|\left(s^{*}\right)^{\prime}\right\|+\left\|g^{\prime}\right\|+\lambda\|s\|\left(T\left\|f^{\prime}\right\|+\|f\|\right)}{\varepsilon} .
$$

This means that

$$
\left|\left(s^{*}\right)^{\prime}\left(t_{1}\right)-\left(s^{*}\right)^{\prime}\left(t_{2}\right)\right| \leq C\left|t_{1}-t_{2}\right| \text { for } t_{1}, t_{2} \in[0, T] .
$$

Proof. Integrating both sides of (9) from 0 to $t$, we obtain:

$$
\varepsilon\left(s(t)-s^{0}\right)=\int_{0}^{t}\left(-s(u)+K^{+} s(u)\right) d u
$$

or equivalently,

$$
s(t)=s^{0}+\frac{1}{\varepsilon} \int_{0}^{t}\left(-s(u)+K^{+} s(u)\right) d u=: L^{+} s(t) .
$$

If a function $s \in C([0, T])$ solves Eq. (27), then it is differentiable, it solves the integrodifferential equation (9), and $s(0)=s^{0}$. Therefore it is sufficient to show that operator $L^{+}$defined in (27) has a unique fixed point. 
By virtue of estimate (15), it follows from (27) that for any two functions $s_{1}, s_{2} \in$ $C([0, T])$,

$$
\begin{aligned}
\left|L^{+} s_{1}(t)-L^{+} s_{2}(t)\right| \leq & \frac{1}{\varepsilon} \int_{0}^{t}\left|s_{1}(u)-s_{2}(u)\right| d u \\
& +\frac{1}{\varepsilon} \int_{0}^{t}\left|K^{+} s_{1}(u)-K^{+} s_{2}(u)\right| d u \\
\leq & \frac{1}{\varepsilon} \int_{0}^{t}\left\|s_{1}-s_{2}\right\| \cdot \lambda\|f\| u d u+\frac{1}{\varepsilon} \int_{0}^{t}\left\|s_{1}-s_{2}\right\| d u \\
= & \frac{1}{\varepsilon}\left\|s_{1}-s_{2}\right\|\left(\lambda\|f\| \frac{t^{2}}{2}+t\right) \\
\leq & \left\|s_{1}-s_{2}\right\| \frac{\frac{\lambda}{2}\|f\| T+1}{\varepsilon} t
\end{aligned}
$$

for $0 \leq t \leq T$. Using the same technique as in the proof of Theorem 1 it can be shown that, for a certain $N$, operator $L^{N}$ is a contraction in the Banach space $C([0, T])$. Therefore operator $L^{+}$has exactly one fixed point $s^{*}(t)$, the unique solution of (9), (10) in $C^{1}([0, T])$.

Since $K^{+} s^{*}(t) \geq 0$, this solution $s^{*}(t)$ is greater than or equal to the solution of initial value problem

$$
\begin{aligned}
\varepsilon s_{c o m p}^{\prime}(t) & =-s_{c o m p}(t), \\
s_{c o m p}(0) & =s^{0}
\end{aligned}
$$

for which we evidently have

$$
s_{\text {comp }}(t)=s^{0} e^{-\frac{1}{\varepsilon} t} \geq 0 .
$$

Hence, $s^{*}(t)$ is also non-negative in $[0, T]$.

The fact that the solution $s^{*}(t)$ is bounded from above by 1 can be verified by indirect proof. If there is any $t_{2} \in(0, T]$ for which

$$
s^{*}\left(t_{2}\right)>1
$$

then due to continuity of the function $s^{*}(t)$ there exists a $t_{1} \in\left(0, t_{2}\right)$ such that

$$
\begin{aligned}
s^{*}\left(t_{1}\right) & =1, \\
s^{*}(t) & >1 \text { for all } t_{1}<t \leq t_{2} .
\end{aligned}
$$

By the mean value theorem, there exists some intermediate point $t_{3} \in\left(t_{1}, t_{2}\right)$, where

$$
\left(s^{*}\right)^{\prime}\left(t_{3}\right)>0 \text {. }
$$

By virtue of (9) and (6),

$$
\varepsilon\left(s^{*}\right)^{\prime}\left(t_{3}\right)=-s^{*}\left(t_{3}\right)+1-\lambda v_{\text {old }}\left(t_{3}\right) \leq-s^{*}\left(t_{3}\right)+1<-1+1=0,
$$

which contradicts $(34)$.

The bound $1 / \varepsilon$ for the derivative is easily obtained from integro-differential equation (9), using (6), (7), and the upper bound for $s(t)$ :

$$
\begin{aligned}
& \varepsilon s^{\prime}(t)=-s(t)+\max (0, K s(t)) \leq-s(t)+1 \leq 1, \\
& \varepsilon s^{\prime}(t) \geq-s(t) \geq-1
\end{aligned}
$$


To show that solution $s^{*}(t)$ satisfies Lipschitz condition of order 1 , we proceed as follows. Let $t_{1}, t_{2} \in[0, T]$. Then by virtue of (9) and Corollary 1 ,

$$
\begin{aligned}
\left|s^{* \prime}\left(t_{1}\right)-s^{* \prime}\left(t_{2}\right)\right| \leq & \frac{1}{\varepsilon}\left|-s^{*}\left(t_{1}\right)+s^{*}\left(t_{2}\right)\right|+\frac{1}{\varepsilon}\left|K^{+} s^{*}\left(t_{1}\right)-K^{+} s^{*}\left(t_{2}\right)\right| \\
\leq & \frac{1}{\varepsilon}\left|s^{*}\left(t_{1}\right)-s^{*}\left(t_{2}\right)\right|+\frac{1}{\varepsilon}\left|K s^{*}\left(t_{1}\right)-K s^{*}\left(t_{2}\right)\right| \\
\leq & \frac{1}{\varepsilon}\left|s^{*}\left(t_{1}\right)-s^{*}\left(t_{2}\right)\right|+\frac{1}{\varepsilon}\left|g\left(t_{1}\right)-g\left(t_{2}\right)\right| \\
& +\frac{\lambda}{\varepsilon}\left|\int_{0}^{t_{1}} f(a) s\left(t_{1}-a\right) d a-\int_{0}^{t_{2}} f(a) s\left(t_{2}-a\right) d a\right| \\
\leq & \frac{1}{\varepsilon}\left(\left\|\left(s^{*}\right)^{\prime}\right\|+\left\|g^{\prime}\right\|\right)\left|t_{1}-t_{2}\right| \\
& +\frac{\lambda}{\varepsilon}\left|\int_{0}^{t_{2}}\left(f\left(t_{1}-a\right)-f\left(t_{2}-a\right)\right) s(a) d a-\int_{t_{1}}^{t_{2}} f\left(t_{1}-a\right) s(a) d a\right| \\
\leq & \frac{1}{\varepsilon}\left(\left\|\left(s^{*}\right)^{\prime}\right\|+\left\|g^{\prime}\right\|\right)\left|t_{1}-t_{2}\right| \\
& +\frac{\lambda}{\varepsilon} T\left\|f^{\prime}\right\|\|s\|\left|t_{1}-t_{2}\right|+\frac{\lambda}{\varepsilon}\|f\|\|s\|\left|t_{1}-t_{2}\right| \\
= & \frac{\left\|\left(s^{*}\right)^{\prime}\right\|+\left\|g^{\prime}\right\|+\lambda\|s\|\left(T\left\|f^{\prime}\right\|+\|f\|\right)}{\varepsilon}\left|t_{1}-t_{2}\right| . \quad \square
\end{aligned}
$$

\section{Numerical approximation.}

4.1. Difference method for the initial value problem. To discretize the problem

$$
\begin{aligned}
\varepsilon s^{\prime}(t) & =-s(t)+\max (0, K s(t)), \quad 0 \leq t \leq T, \\
K s(t) & =g(t)-\lambda \int_{0}^{t} f(a) s(t-a) d a \\
s(0) & =s^{0}
\end{aligned}
$$

using $N$ equal time steps, we set

$$
t_{n}=n \cdot \frac{T}{N}=n \cdot h \quad \text { for } n=0,1,2, \ldots, N .
$$

Let us replace the derivative by a forward difference quotient, and approximate the integral in (40) by the trapezoid rule. Then for $n=0,1,2, \ldots, N-1$,

$$
\begin{gathered}
s^{\prime}\left(t_{n}\right)=\frac{s\left(t_{n+1}\right)-s\left(t_{n}\right)}{h}+\Delta_{n} \\
\int_{0}^{t_{n}} f(a) s\left(t_{n}-a\right) d a=h \sum_{j=0}^{n} a_{j} s\left(t_{j}\right) f\left(t_{n-j}\right)+\bar{\Delta}_{n}
\end{gathered}
$$

where $a_{0}=a_{n}=1 / 2$ and $a_{1}=a_{2}=\cdots=a_{n-1}=1$. 
By Theorem 7 in the Appendix, the error terms $\Delta_{n}$ and $\bar{\Delta}_{n}$ have the estimates

$$
\begin{aligned}
& \left|\Delta_{n}\right| \leq C h, \\
& \left|\bar{\Delta}_{n}\right| \leq \frac{5}{6} C T h^{2},
\end{aligned}
$$

where $C$ is the Lipschitz constant given in (24). Substituting (43) and (44) into integrodifferential equation (39), we obtain

$$
\begin{aligned}
& \varepsilon\left(\frac{s\left(t_{n+1}\right)-s\left(t_{n}\right)}{h}+\Delta_{n}\right) \\
& =-s\left(t_{n}\right)+\max \left(0, g\left(t_{n}\right)-\lambda\left(h \sum_{j=0}^{n} a_{j} f\left(t_{n-j}\right) s\left(t_{j}\right)+\bar{\Delta}_{n}\right)\right), \\
& s(0)=s^{0} .
\end{aligned}
$$

The corresponding difference scheme is

$$
\begin{aligned}
\varepsilon \frac{s_{n+1}-s_{n}}{h} & =-s_{n}+\max \left(0, g\left(t_{n}\right)-\lambda h \sum_{j=0}^{n} a_{j} f\left(t_{n-j}\right) s_{j}\right), \\
s_{0} & =s^{0} .
\end{aligned}
$$

TheOREm 3. The error of difference method (49), (50) for solving initial value problem $(39)-(41)$ is of the order $O(h)$.

Proof. A linear system for the error sequence

$$
e_{n}=s\left(t_{n}\right)-s_{n}
$$

is obtained by subtracting (49) from (47), and (50) from (48):

$$
\begin{gathered}
\varepsilon\left(\frac{e_{n+1}-e_{n}}{h}+\Delta_{n}\right) \\
=-e_{n}+\max \left(0, g\left(t_{n}\right)-\lambda\left(h \sum_{j=0}^{n} a_{j} f\left(t_{n-j}\right) s\left(t_{j}\right)+\bar{\Delta}_{n}\right)\right) \\
-\max \left(0, g\left(t_{n}\right)-\lambda h \sum_{j=0}^{n} a_{j} f\left(t_{n-j}\right) s_{j}\right), \\
e_{0}=0 .
\end{gathered}
$$


Solving (52) for $e_{n+1}$, we get

$$
\begin{aligned}
e_{n+1}= & -h \Delta_{n}+\left(1-\frac{h}{\varepsilon}\right) e_{n} \\
& +\frac{h}{\varepsilon} \max \left(0, g\left(t_{n}\right)-\lambda\left(h \sum_{j=0}^{n} a_{j} f\left(t_{n-j}\right) s\left(t_{j}\right)+\bar{\Delta}_{n}\right)\right) \\
& -\frac{h}{\varepsilon} \max \left(0, g\left(t_{n}\right)-\lambda h \sum_{j=0}^{n} a_{j} f\left(t_{n-j}\right) s_{j}\right) .
\end{aligned}
$$

From (54), the triangle inequality, and Corollary 1 from the Appendix it follows that

$$
\begin{aligned}
\left|e_{n+1}\right| & <\left|1-\frac{h}{\varepsilon}\right|\left|e_{n}\right|+\frac{\lambda h^{2}}{\varepsilon}\left|\sum_{j=0}^{n} a_{j} f\left(t_{n-j}\right) e_{j}\right|-h \Delta_{n}+\frac{\lambda h}{\varepsilon} \bar{\Delta}_{n} \\
& \leq\left(\left|1-\frac{h}{\varepsilon}\right|+\frac{\lambda h^{2}}{2 \varepsilon}|f(0)|\right)\left|e_{n}\right|+\frac{\lambda h^{2}}{\varepsilon} \sum_{j=0}^{n-1} a_{j} f\left(t_{n-j}\right)\left|e_{j}\right|-h \Delta_{n}+\frac{\lambda h}{\varepsilon} \bar{\Delta}_{n} .
\end{aligned}
$$

If we make $h$ small enough, that is if

$$
\begin{aligned}
h \leq 2 \varepsilon & \text { for } f(0)=0, \\
h \leq \min \left(\frac{2}{\lambda f(0)}, 2 \varepsilon\right) & \text { for } f(0)>0,
\end{aligned}
$$

then (55) implies that

$$
\begin{aligned}
\left|e_{n+1}\right| & \leq\left|e_{n}\right|+\frac{\lambda h^{2}}{\varepsilon}\|f\| \sum_{j=0}^{n-1}\left|e_{j}\right|+h \Delta_{n}+\frac{\lambda h}{\varepsilon} \bar{\Delta}_{n} \\
& \leq\left|e_{n}\right|+\frac{\lambda h^{2}}{\varepsilon}\|f\| \sum_{j=0}^{n-1}\left|e_{j}\right|+C h^{2}+\frac{5 \lambda T}{6 \varepsilon} C h^{3} \\
& \leq\left|e_{n}\right|+\frac{\lambda\|f\|}{\varepsilon} h^{2} \sum_{j=0}^{n-1}\left|e_{j}\right|+\left(1+\frac{5 \lambda T}{3}\right) C h^{2} .
\end{aligned}
$$

By virtue of Lemma 2 from the Appendix and (24) it follows from (57) that

$$
\begin{aligned}
\left|e_{n}\right| & \leq n\left(1+\frac{5 \lambda T}{3}\right) C h^{2}\left(1+\sqrt{\frac{\lambda\|f\|}{\varepsilon}} h\right)^{n} \\
& \leq\left(1+\frac{5 \lambda T}{3}\right) C T h \exp \sqrt{\frac{\lambda\|f\|}{\varepsilon}} \\
& =\left(\left\|\left(s^{*}\right)^{\prime}\right\|+\left\|g^{\prime}\right\|+\lambda\|s\|\left(T\left\|f^{\prime}\right\|+\|f\|\right)\right) T \frac{h}{\varepsilon} \exp \sqrt{\frac{\lambda\|f\|}{\varepsilon}} \\
& =O(h) .
\end{aligned}
$$

Thus, the difference method for the initial value problem converges linearly in stepsize $h$. 
4.2. Difference method for the quasi-equilibrium problem. Discretization of the quasiequilibrium problem,

$$
\begin{gathered}
0=-s(t)+\max (0, K s(t)) \\
K s(t)=g(t)-\lambda \int_{0}^{t} f(a) s(t-a) d a
\end{gathered}
$$

with $N$ equal time steps

$$
t_{n}=n \frac{T}{N}=n h \quad \text { for } n=0,1,2, \ldots, N,
$$

yields (here we approximate the integral with the trapezoid rule):

$$
s\left(t_{n}\right)=\max \left(0, g\left(t_{n}\right)-\lambda\left(h \sum_{j=0}^{n} a_{j} f\left(t_{n-j}\right) s\left(t_{j}\right)+\widetilde{\Delta}_{n}\right)\right)
$$

where $a_{0}=a_{n}=1 / 2$ and $a_{1}=a_{2}=\cdots=a_{n-1}=1$. In (62), $\widetilde{\Delta}_{n}$ is an error term, for which Theorem 6 in the Appendix provides the estimate:

$$
\left|\widetilde{\Delta}_{n}\right| \leq \frac{1}{2} C_{q e} T h
$$

with constant $C_{q e}$ explicitly given in (14).

ThEOREM 4. The error of difference method

$$
\begin{aligned}
s_{0} & =\max (0, g(0))=s(0) \\
s_{n+1} & =\max \left(0, g\left(t_{n+1}\right)-\lambda h \sum_{j=0}^{n} a_{j} f\left(t_{n-j}\right) s_{j}\right) \\
\text { for } n & =0,1,2, \ldots,
\end{aligned}
$$

for solving quasi-equilibrium problem (59) is of the order $O(h)$.

Proof. A system for the error sequence

$$
e_{n}=s\left(t_{n}\right)-s_{n}
$$

is obtained by subtracting (65) from (62):

$$
\begin{aligned}
e_{0}= & 0 \\
e_{n+1}= & \max \left(0, g\left(t_{n}\right)-\lambda\left(h \sum_{j=0}^{n} a_{j} f\left(t_{n-j}\right) s\left(t_{j}\right)+\widetilde{\Delta}_{n}\right)\right) \\
& -\max \left(0, g\left(t_{n}\right)-\lambda h \sum_{j=0}^{n} a_{j} f\left(t_{n-j}\right) s_{j}\right) .
\end{aligned}
$$


From the triangle inequality and Corollary 1 it follows that

$$
\begin{aligned}
\left|e_{n+1}\right| & \leq \lambda h \sum_{j=0}^{n}\left|f\left(t_{n-j}\right)\right|\left|e_{j}\right|+\lambda \widetilde{\Delta}_{n} \\
& \leq \lambda h\|f\| \sum_{j=0}^{n}\left|e_{j}\right|+\frac{1}{2} \lambda C_{e q} T h .
\end{aligned}
$$

By Lemma 3 from the Appendix, we obtain the estimate

$$
\left|e_{n}\right| \leq \frac{1}{2} \lambda C_{e q} T h(1+\lambda h\|f\|)^{n} .
$$

The sequence $(1+\lambda h\|f\|)^{n}$ is bounded uniformly in $h$ since

$$
(1+\lambda h\|f\|)^{n}=\left(1+\frac{\lambda\|f\| T}{n}\right)^{n} \uparrow e^{\lambda\|f\| T} \quad \text { as } n \uparrow \infty .
$$

It follows from (71), (72) that

$$
\left|e_{n}\right| \leq \frac{1}{2} \lambda C T e^{\lambda\|f\| T} h=O(h) .
$$

Fig. 1 shows graphs of numerical solutions for a typical initial-value problem and the associated quasi-equilibrium problem. In Fig. 2 the solutions of the initial value problem are compared for different values of the time step $h$. It is not recommended to choose the $h>\varepsilon$, because then instabilities occur.

\section{Asymptotic approximation.}

5.1. Leading order approximation: construction using the boundary function method. Using the boundary function method (see [2], [9], [10]) for singularly perturbed problems, we can represent the uniform asymptotic approximation of the solution of the singularly perturbed initial value problem

$$
\begin{aligned}
\varepsilon s^{\prime}(t) & =-s(t)+\max (0, K s(t)), \quad 0 \leq t \leq T, \\
K s(t) & =g(t)-\lambda \int_{0}^{t} f(a) s(t-a) d a \\
s(0) & =s^{0}
\end{aligned}
$$

in the form

$$
s(t)=\bar{s}(t)+\Pi s\left(\frac{t}{\varepsilon}\right),
$$

where $\bar{s}(t)$ is the regular part, and $\Pi s(t / \varepsilon)$ is the boundary layer part of the approximation. The boundary layer part is necessary to correctly describe the pulse of seedlings in the first few years of forest growth; it decays exponentially to zero as time increases. In what follows, the rescaled time variable of the boundary layer part is denoted by

$$
\tau=t / \varepsilon .
$$




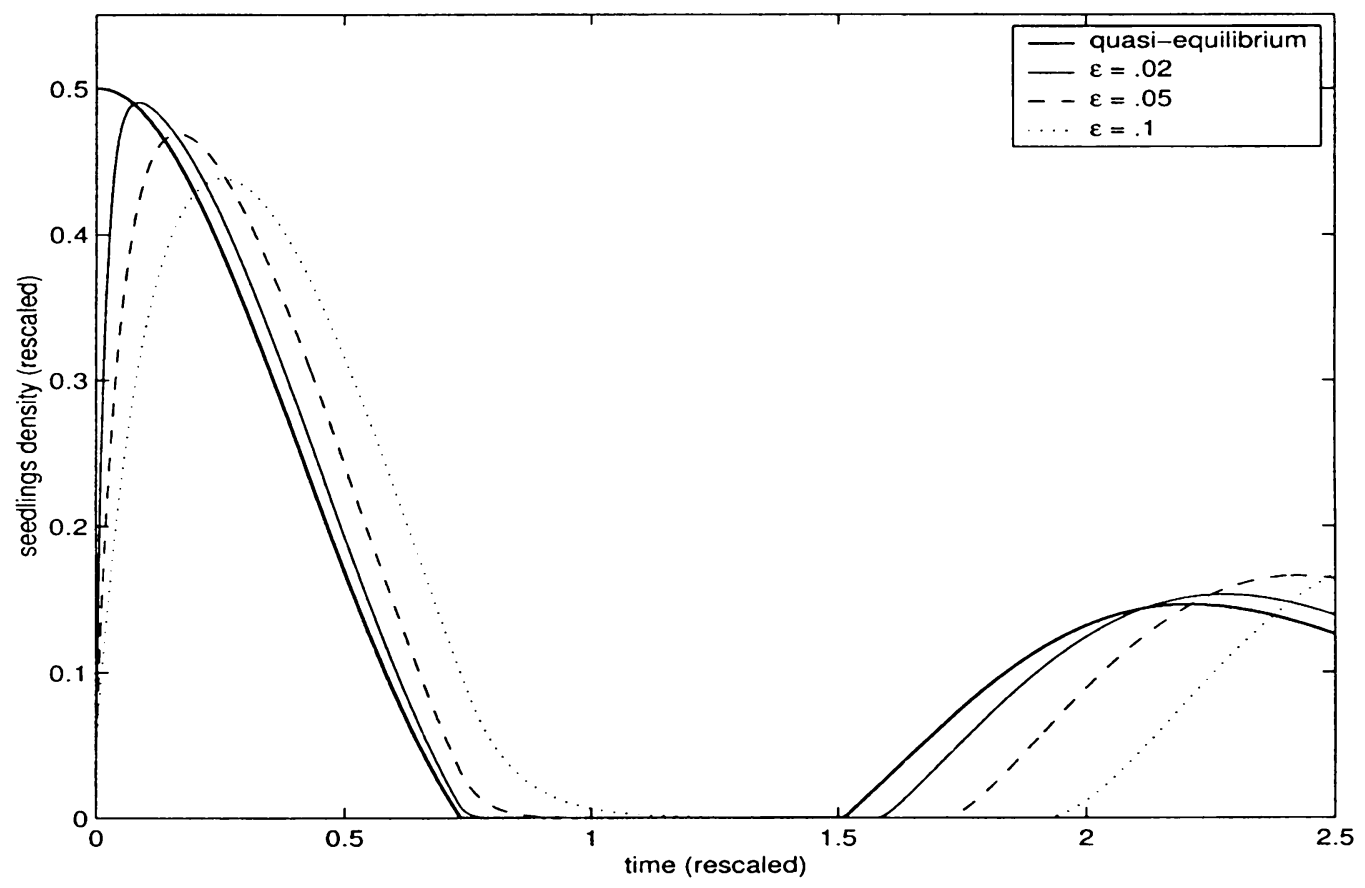

FIG. 1. Numerical solution (stepsize $h=0.005$ ) of initial value problem $\varepsilon s^{\prime}(t)=-s(t)+\max \left(0,1-\lambda v_{\text {old }}(t)-\lambda \int_{0}^{t} s(t-a) a e^{-a} d a\right)$, $s(0)=0.05$, for $\lambda=10, v_{\text {old }}(t)=0.05 t e^{-t}$, and three different values of $\varepsilon$. The quasi-equilibrium solution is shown as a thick line.

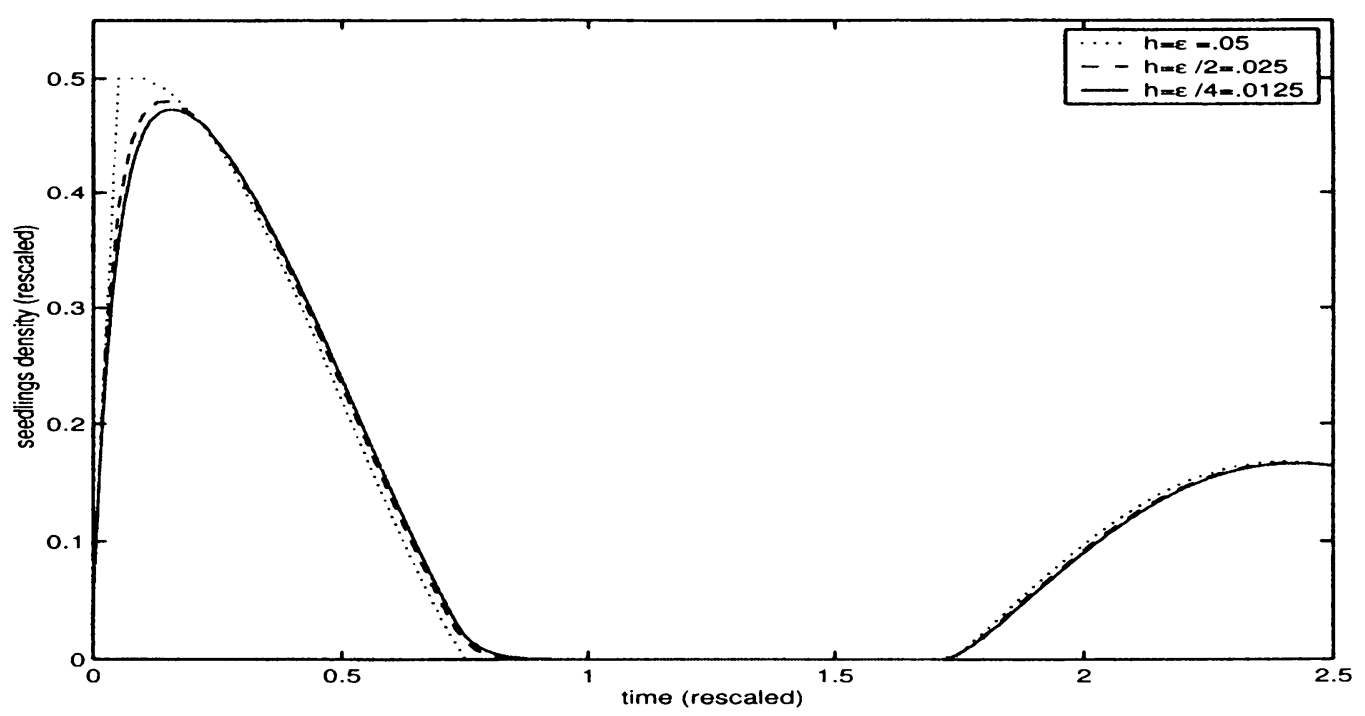

FIG. 2. Numerical solution with different stepsizes for initial value problem $\varepsilon s^{\prime}(t)=-s(t)+\max \left(0,1-\lambda v_{\text {old }}(t)-\lambda \int_{0}^{t} s(t-a) a e^{-a} d a\right)$, $s(0)=0.05$, for $\lambda=10, v_{\text {old }}(t)=0.05 t e^{-t}$, and $\varepsilon=.05$. 
Substitution of representation (77), with (78), into problem (74)--(76) yields

$$
\begin{aligned}
\varepsilon \cdot \frac{d \bar{s}}{d t}+\frac{d \Pi s}{d \tau}= & -\bar{s}(t)-\Pi s(\tau) \\
& +\max \left(0, g(t)-\lambda \int_{0}^{t} f(t-a)\left(\bar{s}(a)+\Pi s\left(\frac{a}{\varepsilon}\right)\right) d a\right), \\
= & -\bar{s}(t)+\max \left(0, g(t)-\lambda \int_{0}^{t} f(t-a) \bar{s}(a) d a\right) \\
& -\Pi s(\tau) \\
& +\max \left(0, g(\varepsilon \tau)-\lambda \int_{0}^{\varepsilon \tau} f(\varepsilon \tau-a)\left(\bar{s}(a)+\Pi s\left(\frac{a}{\varepsilon}\right)\right) d a\right) \\
& -\max \left(0, g(\varepsilon \tau)-\lambda \int_{0}^{\varepsilon \tau} f(\varepsilon \tau-a) \bar{s}(a) d a\right), \\
\bar{s}(0)+\Pi s(0)= & s^{0} .
\end{aligned}
$$

Equation (79) can be split into two equations in variables $t$ and $\tau$, respectively:

$$
\begin{aligned}
\varepsilon \cdot \frac{d \bar{s}}{d t}= & -\bar{s}(t)+\max \left(0, g(t)-\lambda \int_{0}^{t} f(t-a) \bar{s}(a) d a\right) \\
\frac{d \Pi s}{d \tau}= & -\Pi s(\tau) \\
& +\max \left(0, g(\varepsilon \tau)-\lambda \int_{0}^{\varepsilon \tau} f(\varepsilon \tau-a)\left(\bar{s}(a)+\Pi s\left(\frac{a}{\varepsilon}\right)\right) d a\right) \\
& -\max \left(0, g(\varepsilon \tau)-\lambda \int_{0}^{\varepsilon \tau} f(\varepsilon \tau-a) \bar{s}(a) d a\right)
\end{aligned}
$$

To obtain the leading order approximation, we substitute

$$
\begin{aligned}
\bar{s}(t) & =\overline{s_{0}}(t)+O(\varepsilon), \\
\Pi s(\tau) & =\Pi_{0} s(\tau)+O(\varepsilon),
\end{aligned}
$$

into equations (81), (82) and into initial condition (80), and omit terms of order $\varepsilon$ and higher. We obtain the integral equation

$$
0=-\overline{s_{0}}(t)+\max \left(0, g(t)-\lambda \int_{0}^{t} f(t-a) \overline{s_{0}}(a) d a\right)
$$

for the regular part, and the initial value problem

$$
\begin{aligned}
\frac{d \Pi_{0} s}{d \tau} & =-\Pi_{0} s(\tau)+\max (0, g(0))-\max (0, g(0)) \\
& =-\Pi_{0} s(\tau), \\
\Pi_{0} s(0) & =s^{0}-\overline{s_{0}}(0)
\end{aligned}
$$

for the boundary layer part of the leading order asymptotic solution. Integral equation (85) for the regular part is exactly the quasi-equilibrium problem (11). An approximate solution of (85) can be found numerically with difference method (64), (65).

The problem (86), (87) for the boundary layer part has solution

$$
\Pi_{0} s(\tau)=\left(s^{0}-\overline{s_{0}}(0)\right) e^{-\tau} .
$$




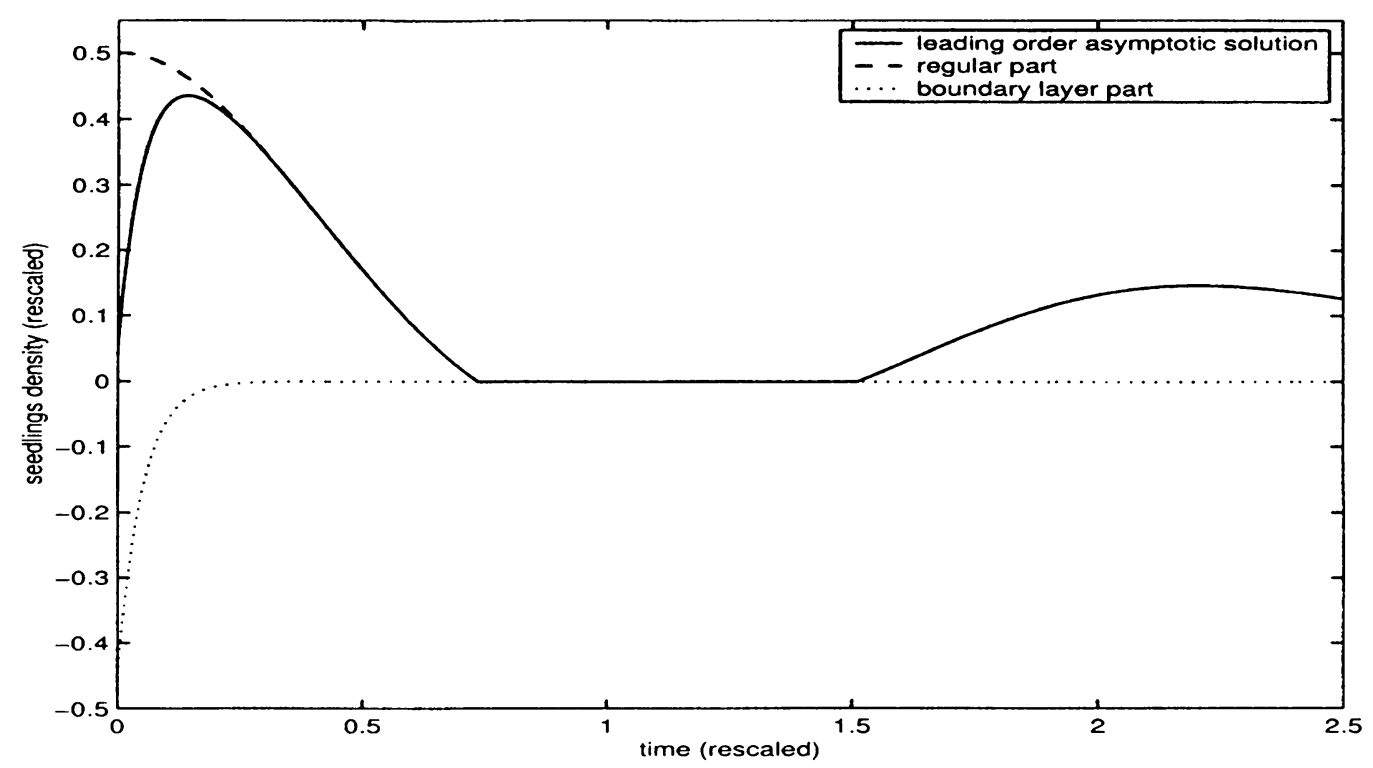

FIG. 3. Leading order asymptotic solution of initial value problem $\varepsilon s^{\prime}(t)=-s(t)+\max \left(0,1-\lambda v_{\text {old }}(t)-\lambda \int_{0}^{t} s(t-a) a e^{-a} d a\right), s(0)=0.05$, for $\lambda=10, v_{\text {old }}(t)=.05 t e^{-t}$, and $\varepsilon=.05$. The asymptotic solution (solid line) is the sum of regular part (quasi-equilibrium solution) and boundary layer part (exponentially decaying to zero).

Fig. 3 illustrates how the asymptotic solution is obtained as a sum of regular and boundary layer parts. Fig. 4 displays asymptotic solution and numerical solution for the same initial value problem $(\varepsilon=.05, h=.005)$.

5.2. Estimation of the remainder. Let us represent the exact solution $s(t)$ of $(74)-(76)$ in the form

$$
s(t)=\overline{s_{0}}(t)+\Pi_{0} s(\tau)+u(t)
$$

where $\overline{s_{0}}(t)$ and $\Pi_{0} s(\tau)$ are the regular and boundary layer parts, respectively, of the leading order approximation constructed in the previous section, and $u(t)$ is the error or, so-called, remainder term. Substituting (89) into the initial value problem (74), (76) we obtain an integro-differential equation for the remainder $u(t)$ :

$$
\begin{aligned}
\varepsilon \frac{d \overline{s_{0}}}{d t}+\frac{d \Pi_{0} s}{d \tau}+ & \varepsilon \frac{d u}{d t}=-\overline{s_{0}}(t)-\Pi_{0} s(\tau)-u(t) \\
& +\max \left(0, g(t)-\lambda \int_{0}^{t} f(t-a)\left(\overline{s_{0}}(a)+\Pi_{0} s\left(\frac{a}{\varepsilon}\right)+u(a)\right) d a\right)
\end{aligned}
$$

with initial condition

$$
\overline{s_{0}}(0)+\Pi_{0} s(0)+u(0)=0
$$




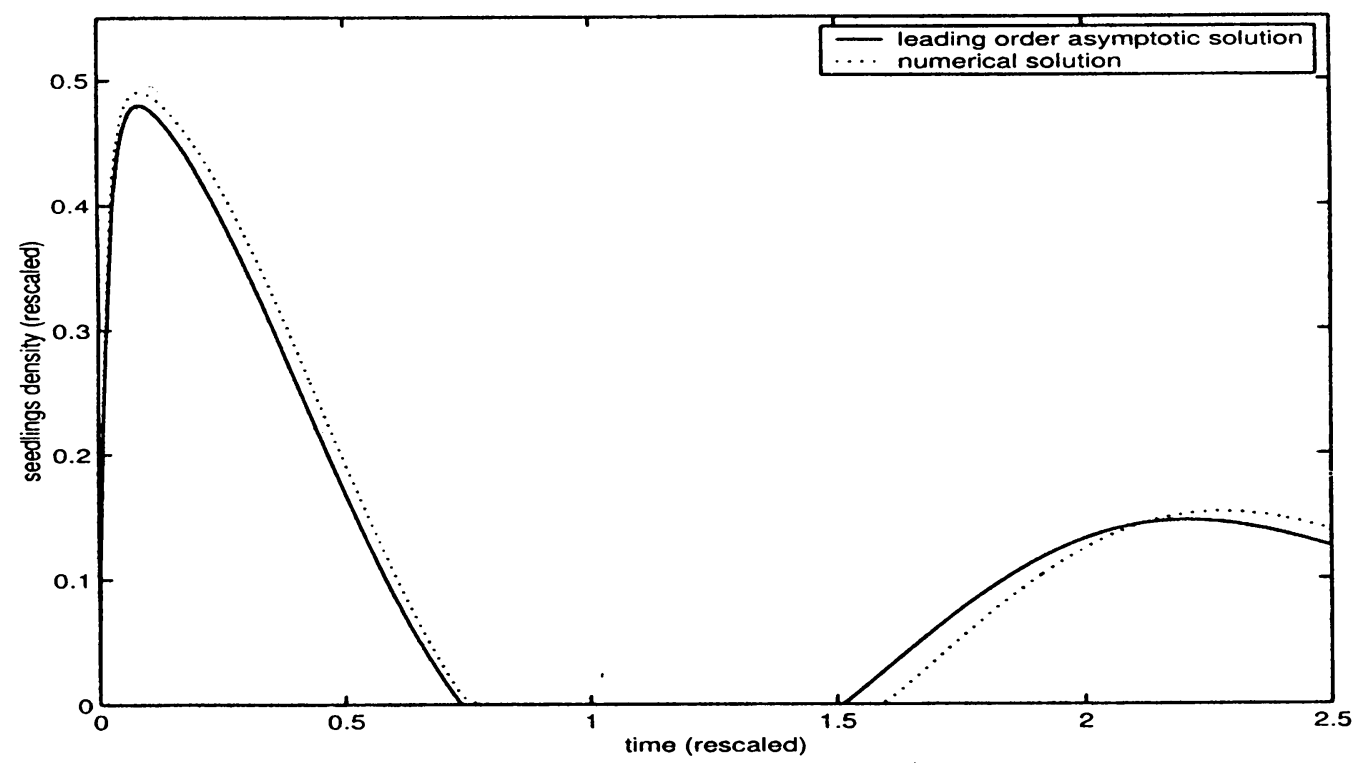

FIG. 4. Comparison of leading order asymptotic solution from Fig. 3 with numerical solution from Fig. $1(\varepsilon=.02, h=.005)$.

By virtue of (85), (86), and (87), the initial value problem (90), (91) simplifies to

$$
\begin{aligned}
\varepsilon \frac{d u}{d t}= & -u(t)+R(u ; t), \\
R(u ; t)= & -\varepsilon \frac{d \overline{s_{0}}}{d t} \\
& +\max \left(0, g(t)-\lambda \int_{0}^{t} f(t-a)\left(\overline{s_{0}}(a)+\Pi_{0} s\left(\frac{a}{\varepsilon}\right)+u(a)\right) d a\right) \\
& -\max \left(0, g(t)-\lambda \int_{0}^{t} f(t-a) \overline{s_{0}}(a) d a\right), \\
u(0)= & 0 .
\end{aligned}
$$

THEOREM 5. The solution of initial value problem (92)-(94) is of order $O(\varepsilon)$.

Proof. Instead of (92), (93) let us consider an equivalent integral equation

$$
u(t)=\frac{1}{\varepsilon} \int_{0}^{t} e^{-\frac{t-a}{\varepsilon}} R(u ; a) d a,
$$

and let us define the operator $J: C([0, T]) \rightarrow C([0, T])$ by

$$
J v(t)=\frac{1}{\varepsilon} \int_{0}^{t} e^{-\frac{t-a}{\varepsilon}} R(v ; a) d a .
$$


We can use Corollary 1 in the Appendix to show that for all $v_{1}, v_{2} \in C([0, T])$ and all $t \in[0, T]$, we have

$$
\begin{aligned}
\mid R\left(v_{1} ; t\right) & -R\left(v_{2} ; t\right) \mid \\
= & \mid \max \left(0, g(t)-\lambda \int_{0}^{t} f(t-a)\left(\overline{s_{0}}(a)+\Pi_{0} s\left(\frac{a}{\varepsilon}\right)+v_{1}(a)\right) d a\right) \\
& -\max \left(0, g(t)-\lambda \int_{0}^{t} f(t-a)\left(\overline{s_{0}}(a)+\Pi_{0} s\left(\frac{a}{\varepsilon}\right)+v_{2}(a)\right) d a\right) \mid \\
\leq & \left|-\lambda \int_{0}^{t} f(t-a) v_{1}(a) d a+\lambda \int_{0}^{t} f(t-a) v_{2}(a) d a\right| \\
= & \lambda\left|\int_{0}^{t} f(t-a)\left(v_{1}(a)-v_{2}(a)\right) d a\right| \\
\leq & \lambda\|f\| t\left\|v_{1}-v_{2}\right\| .
\end{aligned}
$$

Using this estimate we may conclude from (95) that

$$
\begin{aligned}
\left|J v_{1}(t)-J v_{2}(t)\right| & \leq \frac{1}{\varepsilon} \int_{0}^{t} e^{-\frac{t-a}{\varepsilon}}\left|R\left(v_{1} ; a\right)-R\left(v_{2} ; a\right)\right| d a \\
& \leq \frac{\lambda\|f\|}{\varepsilon}\left\|v_{1}-v_{2}\right\| \int_{0}^{t} e^{-\frac{t-a}{\varepsilon}} a d a \\
& =\frac{\lambda\|f\|}{\varepsilon}\left\|v_{1}-v_{2}\right\|\left(\varepsilon t-\varepsilon^{2}\left(1-e^{-\frac{t}{\varepsilon}}\right)\right) \\
& \leq \frac{\lambda\|f\|}{\varepsilon}\left\|v_{1}-v_{2}\right\| \varepsilon t .
\end{aligned}
$$

Using the same technique as in the proof of Theorem 1, it can be shown that operator $J^{N}$ for a certain positive integer $N$ is a contraction with a Lipschitz constant $0<q<1$. It, therefore, has a unique fixed point $u$, which satisfies the estimate

$$
\|u\| \leq \frac{1}{1-q}\left\|J^{N} v_{0}\right\|,
$$

where $v_{0} \in C([0, T])$ is some starting function for the sequence of successive approximations $v_{0}, J v_{0}, J^{2} v_{0}, \ldots$ To show that $\left\|J^{N} v_{0}\right\|$ is of order $O(\varepsilon)$, we use an upper estimate for $R(v ; t)$, where $v \in C([0, T])$. From the definition of $R$ in (93) it follows (by virtue of Corollary 1 and the triangle inequality) that

$$
\begin{aligned}
|R(v ; t)| & \leq \varepsilon\left|\frac{d \overline{s_{0}}}{d t}\right|+\lambda\left|\int_{0}^{t} f(t-a)\left(\Pi_{0} s\left(\frac{a}{\varepsilon}\right)+v(a)\right) d a\right| \\
& \leq O(\varepsilon)+\lambda\|f\|\left|\int_{0}^{t} \Pi_{0} s\left(\frac{a}{\varepsilon}\right) d a\right|+\lambda\|f\|\|v\| t .
\end{aligned}
$$

In this estimate we utilized the fact that the derivative of $\overline{s_{0}}$ is piecewise continuous and therefore bounded. The integral of the boundary layer part $\Pi_{0} s$ is of order $O(\varepsilon)$ because 
by virtue of (88),

$$
\begin{aligned}
\int_{0}^{t} \Pi_{0} s\left(\frac{a}{\varepsilon}\right) d a & =\left(s^{0}-\overline{s_{0}}(0)\right) \int_{0}^{t} e^{-\frac{a}{\varepsilon}} d a \\
& =\left(s^{0}-\overline{s_{0}}(0)\right) \varepsilon\left(1-e^{-\frac{t}{\varepsilon}}\right) \\
& \leq\left(s^{0}-\overline{s_{0}}(0)\right) \varepsilon .
\end{aligned}
$$

From (96) and (98) it follows that

$$
\begin{aligned}
|J v(t)| & \leq \frac{1}{\varepsilon} \sup _{0 \leq a \leq t}|R(v ; a)| \int_{0}^{t} e^{-\frac{t-a}{\varepsilon}} d a \\
& =\sup _{0 \leq a \leq t}|R(v ; a)|\left(1-e^{-\frac{t}{\varepsilon}}\right) \\
& \leq O(\varepsilon)+\lambda\|f\| \int_{0}^{t}|u(a)| d a .
\end{aligned}
$$

Now consider the successive approximations

$$
\begin{aligned}
v_{0} & =0, \\
v_{n+1} & =J v_{n} \quad \text { for } n=0,1,2,3, \ldots .
\end{aligned}
$$

Using (100), we obtain the recursion

$$
\begin{aligned}
\left|v_{0}(t)\right| & =0 \\
\left|v_{n+1}(t)\right| & \leq C \varepsilon+\lambda\|f\| \int_{0}^{t}\left|v_{n}(a)\right| d a \text { for } n=0,1,2,3, \ldots,
\end{aligned}
$$

where $C$ is a suitable positive constant independent of $\varepsilon$. By induction we can prove the inequality

$$
\left|v_{n}\right| \leq C \varepsilon \sum_{j=0}^{n-1} \frac{(\lambda\|f\| t)^{j}}{j !} \quad \text { for } n=1,2,3, \ldots
$$

Indeed, (105) is true for $n=1$ since from (103), we have

$$
\left|v_{1}(t)\right| \leq C \varepsilon \text {. }
$$

Assume that (105) is satisfied for $j=1,2, \ldots, n$ for a certain $n$. Then, by recursion $(104)$,

$$
\begin{aligned}
\left|v_{n+1}(t)\right| & \leq C \varepsilon+\lambda\|f\| \int_{0}^{t} C \varepsilon \sum_{j=0}^{n-1} \frac{(\lambda\|f\| a)^{j}}{j !} d a \\
& =C \varepsilon+C \varepsilon \lambda\|f\| \sum_{j=0}^{n-1} \frac{(\lambda\|f\|)^{j}}{j !} \int_{0}^{t} a^{j} d a \\
& =C \varepsilon+C \varepsilon \lambda\|f\| \sum_{j=0}^{n-1} \frac{(\lambda\|f\|)^{j}}{j !} \frac{t^{j+1}}{j+1} \\
& =C \varepsilon \sum_{j=0}^{n} \frac{(\lambda\|f\| t)^{j}}{j !} .
\end{aligned}
$$


Thus, (105) is true for $n+1$, and the induction over $n$ is completed. A consequence of (105) is that

$$
\begin{aligned}
\left|v_{n}(t)\right| & \leq C \varepsilon \sum_{j=0}^{\infty} \frac{(\lambda\|f\| t)^{j}}{j !} \\
& =C \varepsilon e^{\lambda\|f\| t} \\
& \leq C \varepsilon e^{\lambda\|f\| T}
\end{aligned}
$$

Thence,

$$
\left\|v_{n}\right\| \leq C \varepsilon e^{\lambda\|f\| T}=O(\varepsilon)
$$

So, in particular,

$$
\left\|J^{N} v_{0}\right\|=\left\|v_{N}\right\|=O(\varepsilon)
$$

From (98) it then follows that

$$
\|u\|=O(\varepsilon)
$$

6. Conclusion. Utilizing the Lipschitz continuity of the integro-differential equation, we were able to show existence and uniqueness for both the initial value problem and the quasi-equilibrium problem, to prove linear convergence of the numerical scheme, and to estimate the remainder of the asymptotic approximation obtained using the boundary function method. Error bounds were provided, which depend on the small parameter $\varepsilon$. Thus, a prerequisite has been fulfilled for data fitting with nonlinear least squares methods. Also, the methods presented in this paper can be used to study the long-term behavior of the age structure of a forest, i.e., convergence to an equilibrium state, or oscillations.

The boundary function method was earlier used for analysis of somewhat different singularly perturbed integro-differential equations (see, e.g., [9]). Other asymptotic methods ([5], [6], [8]) can be applied for construction of the asymptotic solution as well.

We also need to mention that the analysis presented in this paper will work for a more general statement of the problem:

$$
\varepsilon s^{\prime}(t)=-s(t)+\max (0, H(\lambda, v(t)))
$$

with a positive nonlinear function $H(\lambda, v(t))$, expression for $v(t)$ given by $(2)$, and initial condition (3). Here we have chosen the form of equation (1) for analysis to simplify technical details and to make the exposition more accessible for a general reader.

\section{Appendix.}

LEMMA 1. For all real numbers $x, y$, the following inequality holds:

$$
\max (0, x)-\max (0, y) \leq \max (0, x-y) \text {. }
$$

Proof. If $x>0$ and $y>0$, then

$$
\max (0, x)-\max (0, y)=x-y \leq \max (0, x-y) .
$$

If $x \leq 0$ and $y \leq 0$, then

$$
\max (0, x)-\max (0, y)=0-0=0 \leq \max (0, x-y) .
$$


If $x>0$ and $y \leq 0$, then

$$
\max (0, x)-\max (0, y)=x-0=x \leq x-y \leq \max (0, x-y) .
$$

If $x \leq 0$ and $y>0$, then

$$
\max (0, x)-\max (0, y)=-y<0 \leq \max (0, x-y) .
$$

In all four cases, the claim (113) was verified.

Corollary 1 . For all real numbers $x, y$, the following inequality holds:

$$
|\max (0, x)-\max (0, y)| \leq|x-y| \text {. }
$$

Proof. By Lemma 1,

$$
\max (0, x)-\max (0, y) \leq \max (0, x-y) \leq|x-y|
$$

and hence- by interchanging $x$ and $y-$

$$
\max (0, y)-\max (0, x) \leq|y-x|=|x-y| .
$$

From (119) and (120) the claim in (118) follows.

LEMMA 2. If a sequence of real numbers $\left(y_{0}, y_{1}, y_{2}, y_{3}, \ldots\right)$ satisfies

$$
\begin{aligned}
y_{0} & =0 \\
\left|y_{1}\right| & \leq C \\
\left|y_{2}\right| & \leq 2 C \\
\left|y_{n+1}\right| & \leq\left|y_{n}\right|+B \sum_{k=1}^{n-1}\left|y_{k}\right|+C \quad \text { for } n=2,3, \ldots
\end{aligned}
$$

with positive constants $B$ and $C$, then the following estimate holds:

$$
\left|y_{n}\right| \leq n C(1+\sqrt{B})^{n} \quad \text { for } n=0,1,2, \ldots \text {. }
$$

Proof by induction. From the assumptions it is evident that the inequality in (125) holds for $n=0,1,2$. Suppose that (125) holds for $n=0,1,2, \ldots, N$, where $N$ is greater than or equal to 2. By substitution of these inequalities into recursion (124), we obtain an estimate for $y_{N+1}$ :

$$
\begin{aligned}
\left|y_{N+1}\right| & \leq\left|y_{N}\right|+B \sum_{k=1}^{N-1}\left|y_{k}\right|+C \\
& \leq N C(1+\sqrt{B})^{N}+B \sum_{k=1}^{N-1} k C(1+\sqrt{B})^{N}+C .
\end{aligned}
$$

To complete the induction step it is sufficient to show that

$$
\left|y_{N+1}\right| \leq N C(1+\sqrt{B})^{N}+B \sum_{k=1}^{N-1} k C(1+\sqrt{B})^{k}+C \leq(N+1) C(1+\sqrt{B})^{N+1} .
$$

Let us divide this inequality by $C$, and use the notation

$$
x=1+\sqrt{B}
$$


to obtain an equivalent inequality

$$
N x^{N}+(x-1)^{2} \sum_{k=1}^{N-1} k x^{k}+1 \leq(N+1) x^{N+1} .
$$

To prove (129), we start with the true inequality

$$
0 \leq 2 x^{N}+2 \sum_{k=0}^{N-1} x^{k}
$$

which is equivalent to

$$
(N-1) x^{N}-\sum_{k=1}^{N-1} x^{k} \leq(N+1) x^{N}+\sum_{k=0}^{N-1} x^{k} .
$$

On account of the identities

$$
\begin{aligned}
(x-1) \sum_{k=1}^{N-1} k x^{k} & =(N-1) x^{N}-\sum_{k=1}^{N-1} x^{k}, \\
\frac{(N+1) x^{N+1}-N x^{N}-1}{x-1} & =(N+1) x^{N}+\sum_{k=0}^{N-1} x^{k},
\end{aligned}
$$

(131) can be written as

$$
(x-1) \sum_{k=1}^{N-1} k x^{k} \leq \frac{(N+1) x^{N+1}-N x^{N}-1}{x-1} .
$$

By multiplication with $x-1$ and rearrangement of terms, (129) follows, and the induction proof is completed.

LEMmA 3. If a sequence of real numbers $\left(y_{0}, y_{1}, y_{2}, y_{3}, \ldots\right)$ satisfies

$$
\begin{aligned}
\left|y_{0}\right| & \leq C \\
\left|y_{n+1}\right| & \leq B \sum_{k=0}^{n}\left|y_{k}\right|+C \quad \text { for } n=1,2, \ldots
\end{aligned}
$$

with positive constants $B, C$, then the following estimate holds:

$$
\left|y_{n}\right| \leq C(B+1)^{n} \quad \text { for } n=0,1,2, \ldots
$$

Proof by induction. For $n=0$ the claim (137) is true because of assumption (135). Assume now that it is also true for every $j=0,1,2, \ldots, n$. Then, by recursion (136), it 
follows for $n+1$ that

$$
\begin{aligned}
\left|y_{n+1}\right| & \leq B \sum_{k=0}^{n}\left|y_{k}\right|+C \\
& \leq B \sum_{k=0}^{n} C(B+1)^{k}+C \\
& =C\left(B \sum_{k=0}^{n}(B+1)^{k}+1\right) \\
& =C\left(B \frac{1-(B+1)^{n+1}}{1-(B+1)}+1\right) \\
& =C(B+1)^{n+1} .
\end{aligned}
$$

THEOREM 6. Let $p(x)$ be a real-valued function, satisfying a Lipschitz condition of order 1 with Lipschitz constant $C>0$ on the interval $[a, b]$, that is

$$
|p(y)-p(x)| \leq C|y-x| \text { for all } x, y \in[a, b]
$$

Then

(a)

$$
\begin{gathered}
\left|\int_{a}^{b} p(t) d t-\frac{b-a}{2}(p(a)+p(b))\right|<\frac{C}{2}(b-a)^{2} \\
\left|\int_{a}^{b} p(t) d t-\frac{b-a}{n}\left(\frac{p(a)+p(b)}{2}+\sum_{k=1}^{n-1} p\left(a+k \frac{b-a}{n}\right)\right)\right|<\frac{C}{2} \frac{(b-a)^{2}}{n} \\
\text { for } n=2,3,4, \ldots
\end{gathered}
$$

Proof of (a).

$$
\begin{array}{rl}
\mid \int_{a}^{b} & p(t) d t-\frac{b-a}{2}(p(a)+p(b)) \mid \\
\quad & \left|\frac{1}{2} \int_{a}^{b}(p(t)-p(a)) d t+\frac{1}{2} \int_{a}^{b}(p(t)-p(b)) d t\right| \\
& \leq \frac{1}{2} \int_{a}^{b}|p(t)-p(a)| d t+\frac{1}{2} \int_{a}^{b}|p(t)-p(b)| d t \\
& \leq \frac{1}{2} \int_{a}^{b} C(t-a) d t+\frac{1}{2} \int_{a}^{b} C(b-t) d t \\
& =\frac{C}{2}(b-a)^{2} .
\end{array}
$$

Proof of (b). Apply (a) to the integrals on the subintervals $\left(a+k \frac{b-a}{N}, a+(k+1) \frac{b-a}{N}\right)$ for $k=0,1,2, \ldots, N-1$. Then sum over $k$.

THEOREM 7. Let $p(x)$ be a real-valued function, continuously differentiable on the interval $[a, b]$, and let the derivative $p^{\prime}(x)$ satisfy Lipschitz condition of order 1 with Lipschitz constant $C>0$, that is

$$
\left|p^{\prime}(y)-p^{\prime}(x)\right| \leq C|y-x| \text { for all } x, y \in[a, b] .
$$


Then

(a) $\left|\frac{p(y)-p(x)}{y-x}-p^{\prime}(x)\right|<C(y-x) \quad$ for $a \leq x<y \leq b$,
(b) $\left|\int_{a}^{b} p(t) d t-\frac{b-a}{2}(p(a)+p(b))\right|<\frac{5 C}{6}(b-a)^{3}$,
(c) $\left|\int_{a}^{b} p(t) d t-\frac{b-a}{n}\left(\frac{p(a)+p(b)}{2}+\sum_{k=1}^{n-1} p\left(a+k \frac{b-a}{n}\right)\right)\right|<\frac{5 C}{6} \frac{(b-a)^{3}}{n^{2}}$

for $n=2,3,4, \ldots$.

Proof of (a). For any $0 \leq x<y \leq T$, we know by the mean value theorem that there exists some $\xi \in(x, y)$ such that

$$
\frac{p(y)-p(x)}{y-x}=p^{\prime}(\xi)
$$

By (141), we have the estimate

$$
\left|p^{\prime}(\xi)-p^{\prime}(x)\right| \leq C(\xi-x)
$$

Substitution of (143) into (144) yields the result:

$$
\left|\frac{p(y)-p(x)}{y-x}-p^{\prime}(x)\right|=\left|p^{\prime}(\xi)-p^{\prime}(x)\right| \leq C(\xi-x)<C(y-x) .
$$

Proof of (b). Let $t \in(a, b]$. Application of part (a) with $x=a$ and $y=t$ yields:

$$
\left|\frac{p(t)-p(a)}{t-a}-p^{\prime}(a)\right|<C(t-a) .
$$

After we multiply (146) by $t-a$ and use the triangle inequality, we see that

$$
p(t)=p(a)+(t-a) p^{\prime}(a)+\theta_{t} C(t-a)^{2}
$$

for some $\theta_{t} \in[-1,1]$, which depends on $t$. In particular, for $t=b$,

$$
p(b)=p(a)+(b-a) p^{\prime}(a)+\theta_{b} C(b-a)^{2} .
$$

Solving (148) for $p^{\prime}(a)$ and substituting into (147), we obtain:

$$
p(t)=\left(1-\frac{t-a}{b-a}\right) p(a)+\frac{t-a}{b-a} p(b)+C\left(\theta_{t}(t-a)^{2}-\theta_{b}(b-a)(t-a)\right) .
$$

Integrating with respect to $t$ from $a$ and $b$, we get

$$
\int_{a}^{b} p(t) d t=\frac{b-a}{2}(p(a)+p(b))+C \int_{a}^{b}\left(\theta_{t}(t-a)^{2}-\theta_{b}(b-a)(t-a)\right) d t .
$$


Thus

$$
\begin{aligned}
& \left|\int_{a}^{b} p(t) d u-\frac{b-a}{2}(p(a)+p(b))\right| \\
& \quad=C\left|\int_{a}^{b}\left(\theta_{t}(t-a)^{2}-\theta_{b}(b-a)(t-a)\right) d t\right| \\
& \quad \leq C\left(\int_{a}^{b}(t-a)^{2} d t+(b-a) \int_{a}^{b}(t-a) d t\right) \\
& \quad=\frac{5}{6} C(b-a)^{3} .
\end{aligned}
$$

Proof of (c). Apply (b) to the integrals on the subintervals $\left(a+k \frac{b-a}{N}, a+(k+1) \frac{b-a}{N}\right)$ for $k=0,1,2, \ldots, N-1$. Then sum over $k$.

\section{REFERENCES}

[1] J. A. Cochran, The analysis of linear integral equations, McGraw-Hill, New York, 1972.

[2] L. V. Kalachev, Asymptotic methods: application to reduction of models, Natural Resource Modeling 13 (2000), 305-338.

[3] J. Kevorkian and J. D. Cole, Perturbation Methods in Applied Mathematics, Springer-Verlag, New York, 1981.

[4] M. A. Kraemer, L. V. Kalachev, and D. W. Coble, A Class of Models Describing Age Structure Dynamics in a Natural Forest, Natural Resource Modeling, to appear.

[5] G. McFadden and C. D. Oliver, Three-dimensional forest growth model relating tree size, tree number, and stand age: Relation to previous growth models and to self-thinning, Forest Science 34 (1988), 662-676.

[6] R. E. O'Malley, Singular Perturbations Methods for Ordinary Differential Equations, SpringerVerlag, New York, 1991.

[7] C. D. Oliver and B. C. Larson, Forest Stand Dynamics, McGraw-Hill, New York, 1990.

[8] D. R. Smith, Singular-Perturbation Theory, Cambridge University Press, Cambridge, 1985.

[9] A. B. Vasil'eva and V. F. Butuzov, Asymptotic behavior of the solution of an integro-differential equation with a small parameter multiplying the derivative (in Russian), Zhurnal Vychislitel'noi' Matematiki i Matematicheskoi' Fiziki 4 (1964), 183-191.

[10] A. B. Vasil'eva, V. F. Butuzov, and L. V. Kalachev, The Boundary Function Method for Singular Perturbation Problems, SIAM, Philadelphia, 1995. 